\title{
Evaluación del profesor universitario: ¿A mayor categoría profesional mejor profesor? ${ }^{1}$
}

\section{University Professor's evaluation: Does a higher category make a better professor?}

Sonia Casillas Martín ', Marcos Cabezas González , Ana María Pinto Llorente 2

'Facultad de Educación, Universidad de Salamanca, España. \{scasillasma, mcabezasgo\}@usal.es

2Facultad Educación, Universidad Pontificia de Salamanca, España. ampintoll@upsa.es

Resumen

El artículo analiza los resultados de la evaluación docente realizada por los alumnos de la Universidad Pontificia de Salamanca. La metodología seguida es cuantitativa mediante un cuestionario validado con una muestra piloto de 1.200 cuestionarios obtenidos por muestreo aleatorio estratificado. La muestra de profesores que han sido evaluados por los alumnos es de 177 profesores en 257 materias impartidas. Los resultados ponen de manifiesto la utilidad de la evaluación por los alumnos, dado que son los mejores observadores del proceso de enseñanza- aprendizaje, aunque pueden darse sesgos debido a diversos factores, como puede ser la categoría profesional del profesor, variable que estudiamos en este trabajo. Así, las percepciones de los 4.198 alumnos destacan que en algunos aspectos influye la categoría profesional del profesor para considerarle mejor o peor profesor.
Palabras Clave:

Evaluación; Universidad; Evaluación de la docencia universitaria; Calidad.

\begin{abstract}
This article analyses the results from the professor's evaluation that the students of the Universidad Pontificia de Salamanca made. The methodology used is quantitative and the tool that has been used is a questionnaire applied to 1.200 people and obtained by stratified sampling. 177 professors have been evaluated in 257 different subjects. The results show the importance of the evaluation made by the students since they are the best observers of the learning-teaching process although there can be a bias due to diverse issues like the professor's professional category which is the specific topic that we analyze in this research. The perceptions of 4.198 students show that the professional category has a great influence in considering professors better or worse professionals
\end{abstract}




\section{Introducción}

Con este artículo se pretende observar la existencia de diferencias en los resultados de la evaluación docente por los alumnos en la Universidad Pontificia de Salamanca (UPSA), teniendo en cuenta la categoría profesional del profesor.

Dos son los argumentos fundamentales que resaltan la importancia de estos procesos de la evaluación de la docencia del profesor universitario.

El primero, como pone de manifiesto Rodríguez Espinar (1998: 211-212) es que se están produciendo numerosos cambios en la Educación Superior, en los que es clave la evaluación de la docencia. Algunas de estas transformaciones pueden ser la existencia de puntos débiles en la enseñanza universitaria como consecuencia de la expansión, la internalización, el aumento de los costes de acceso, la exigencia de nuevas funciones $u$ obligaciones..., estos y otros cambios hacen surgir la necesidad de la evaluación de la docencia para mejorar el servicio que se ofrece a los alumnos.

Además, y en la misma línea, De Miguel (2003) incide en la importancia de la evaluación docente que se ha multiplicado por el amplio desarrollo y difusión de esta en los últimos años. Señala una serie de datos que avalan esta premisa como "el incremento del número de trabajos realizados, apertura a otros campos profesionales, desarrollo del marco teórico y metodológico, difusión de un vocabulario específico y globalización de los aspectos socioprofesionales". Y este período de expansión provoca una serie de consecuencias como: "nuevas demandas, nuevos clientes, nuevos roles y nuevas tecnologías" (pp. 1-3). El segundo argumento viene dado en función de la utilidad de los resultados obtenidos. Zabalza (1990) para demostrar esta utilidad resalta para qué se utilizan los resultados obtenidos y las distintas formas de utilizar los resultados de la evaluación docente:

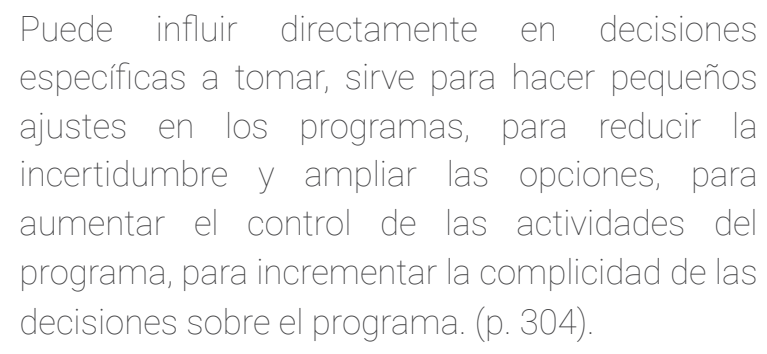

Asimismo, la necesidad y utilidad de la evaluación docente va recobrando cada vez más importancia. De Miguel (1998: 240) considera que dicha evaluación instituye un instrumento ineludible para conseguir los objetivos marcados. Entendemos que es útil porque es provechoso para el fin señalado.

Tejedor (2003) manifiesta que la importancia viene por el papel que desempeña la evaluación de la docencia universitaria. Este es doble: como instrumento técnico de control interno de responsabilidad y de imputabilidad: la Universidad es una realidad que implica una relación entre personas, una estructura, tareas, presupuestos, así como una obligación contractual de ofrecer un servicio de calidad a la clientela.

como un instrumento de formación de intelectuales, de profesionales, en respeto a su misión de sociedad, que es un proyecto intelectual integrado que hace que la universidad sea más 
que la aglomeración de personas y de campos específicos... (pp. 158-159).

Por otro lado, la Agència per a la Qualitat del Sistema Universitari a Catalunya (2002: 6- 16) considera que la evaluación de la actividad docente del profesorado universitario es básica, puesto que se trata de una pieza esencial del sistema universitario, como así se expone en el artículo 45. 3 de la LRU. Además, añade que la importancia de la evaluación de la docencia está en que los resultados obtenidos en esta evaluación sean relevantes para el desarrollo profesional del profesor. Estima que para que el profesor sea participe de este proceso y no lo considere un papeleo más de su labor, el feecback recibido de la evaluación tiene que suponer consecuencias importantes para su desarrollo profesional.

Como ya hemos señalado en otros trabajos relacionados con el tema (Casillas Martín, S. (2006), Casillas Martín, S. (2007), Casillas Martín, S. (2008), Casillas Martín, Sonia y Cabezas González, Marcos. (2009), Casillas Martín, Sonia y Cabezas González, Marcos. (2010)) la evaluación docente del profesorado está íntimamente relacionada con la mejora de la calidad de dicha docencia. Destacando que los resultados obtenidos en las evaluaciones docentes sean cruciales para la mejora del desarrollo profesional del docente.

Son diversos los estudios que tratan el tema de la evaluación del profesorado, de los estudiantes... en el Sistema Universitario (GilFlores, J. (2012), Cano, E. y Ion, G. (2012), Tejedor (2009), etc.). Todos estos autores de prestigio llegan a conclusiones similares a las que abordamos en este trabajo, no dudando en los beneficios y ventajas de la evaluación en la Universidad.

Sin embargo, también existen problemas o inconvenientes en este proceso de evaluación docente, ya que esta, hoy por hoy, sigue siendo un enigma con muchas limitaciones tanto de índole teórico como práctico. Por lo que se observa en este proceso una amplia diversidad de finalidades, así como la carencia de un modelo de buen profesor. La elección de la estrategia evaluativa adecuada puede suponer una contrariedad, puesto que su validez ha de establecerse indirectamente (Tejedor y García-Valcárcel, 1996: 94).

En la mayoría de las ocasiones el problema surge cuando los implicados en la evaluación de la función docente no la perciben como una estrategia o instrumento para la mejora de su actividad. Lo que ocurre en muchas instituciones en las que se evalúa la actuación docente de sus profesores es que ni estos, ni los alumnos, ni las autoridades, lo consideran un instrumento con utilidad para la reforma de la enseñanza. Por otra parte, la evaluación no puede ser considerada en sentido estricto algo que concierne solo al profesorado, sino que incluye a toda la institución universitaria. En este amplio sentido se incluiría la contratación del personal, así como su promoción y evaluación. Resulta contradictorio que desde la Universidad surjan iniciativas de evaluación del profesorado sin que, de forma previa, se haya incidido en aspectos relevantes como la formación y selección del profesorado universitario. Las opiniones ante la utilidad 
son dispersas, la más generalizada es que solamente es útil para las autoridades, ya que disponen de una información "privada" que pueden utilizar según su conveniencia. Se puede afirmar que la falta de utilidad de la evaluación de la función docente del profesorado universitario ha causado daños en las actitudes de los implicados (De Miguel, 1998: 238- 240).

Otro de los problemas es encontrar un criterio aceptable para medir la docencia y posteriormente que tengan la validez suficiente para emitir juicios de valor. Así, Mateo (1990: 319) considera que el mayor inconveniente de cualquier proceso evaluativo es el de encontrar un criterio válido y con una aceptación que tenga un consentimiento común para poder emitir juicios de valor. Concretamente, en el caso de la evaluación de la docencia "determinar el criterio evaluador constituye una ardua tarea, que exige previamente enunciar cuál es su función, hecho que indudablemente va unido a las expectativas discentes de la sociedad a la que va dirigida su labor". Una de las mayores equivocaciones del proceso de evaluación de la docencia universitaria ha sido considerar la subida salarial como un factor motivacional. Porque la mayoría de las personas que se dedican a la docencia universitaria la eligen por otros motivos, como la sensibilidad hacia gratificaciones intrínsecas, este autor indica: "la enseñanza, el contacto con el mundo de las ideas, la investigación, el intercambio intelectual con colegas, o la necesidad de reflexión" (Guerrero y Vicente, 1999: 580).
Sin embargo, a partir del proceso de evaluación de la docencia, parece que se parte del simple supuesto de que, solamente con conocer los resultados obtenidos, se produce la mejora en la enseñanza, es decir, los profesores cuando ven las valoraciones de sus alumnos tenderán a cambiar aquellos aspectos en los que les han evaluado peor. Este supuesto parece ser candoroso, pues nada hace suponer que el hecho de conocer los resultados de la evaluación produzca cambios dirigidos a la mejora en la actuación del profesor. La mejora lleva consigo un proceso mucho más complejo que contiene "toda una serie de condiciones a la planificación y desarrollo del proceso de evaluación". Por ello, es importante aclarar en el proceso de evaluación una serie de aspectos, tales como: "Qué tipo de enseñanza funciona bien, qué habilidades, condiciones, actuaciones... son precisas para desarrollarla y cómo se podrían aplicar a nuestro contexto, cuál es mi posición con respecto a dichos aspectos, qué caminos tengo para mejorar esos puntos débiles" (Zabalza, 1990: 310-312). También, Tejedor (2003: 159) cree que el fin de toda actividad evaluadora debe ser el de mejorar la actividad profesional del profesor universitario. Pero para asegurar el éxito de esta acción o proceso de evaluación, este debe ser útil para todos los implicados, viable en el tiempo, en el espacio y a partir de las condiciones físicas, administrativas, académicas, exacto, en términos de utilizar fuentes de información e instrumentos precisos, objetivo, descriptivo y potencialmente transformador y ético, siendo 
transparente y respetuoso con los valores

Otros estudios vinculan la eficacia del docente únicamente al rendimiento de los alumnos, lo que supone un gran reduccionismo e inconveniente de la evaluación de la labor docente. A propósito de este reduccionismo, Tascón (1998: 818), considera que simplificar la evaluación a la función del profesor sería un inconveniente de este complejo proceso. Jornet (1993: 72- 80) expone que la dificultad de la evaluación del profesorado se corresponde con la de cualquier enfoque institucional. Los planteamientos que definen la evaluación pueden ser demasiado complejos, su uso será correcto si son convenientemente tratados desde modelos estadísticos explicativos o causales. Si no es así, nos puede conducir a pseudoevaluaciones, es decir, a realizar unas interpretaciones de los resultados erróneas o ambiguas, que no se correspondan con la realidad.

Por ello, la utilización de los resultados obtenidos en las evaluaciones, aunque parezca que no pueden perjudicar al individuo objeto de evaluación, se considera que puedan ser dificultosos por afectar a las situaciones básicas de desarrollo institucional y social, y por tanto, también a los individuos. Lo que puede conducir a decisiones que agraven la discriminación entre los profesores evaluados. Esto implica que no es ni oportuno, ni provechoso, ni decente, sino que, en todo caso, se debe apostar por orientar esfuerzos hacia la calidad. Para ello es necesario adquirir cierto grado de responsabilidad por las personas implicadas en la investigación básica y metodológica de la educación superior. Así como en la revisión de los procesos e indicadores para su evaluación. Del mismo modo los problemas que surgen en el proceso de la evaluación de la docencia del profesor universitario, se podrían prever con una fundamentación teórica. Esto es de gran ayuda para determinar aspectos como "el planteamiento de hipótesis, la selección de unidades de análisis e identificación de variables, el diseño, la instrumentalización, análisis, estrategias de utilización de la información, etc. De este modo, tomar como punto de partida un modelo de referencia, definido desde cualquier orientación, permite establecer estrategias de evaluación mejor contextualizadas, en definitiva, más válidas". Otro inconveniente pueden ser las quejas de los profesores, porque ello supone que no están comprometidos para afrontar el cambio. Zabalza recoge como queja más frecuente de los profesores acerca de la evaluación de su función docente: "que nos evalúen pero cuando evalúen todo: ni la Administración, ni las propias instituciones están legitimadas para evaluar a los profesores hasta que ellas mismas no evalúen su funcionamiento y las condiciones en las que actúan aquellos a los que se evalúa" (1990: 314). Desde una perspectiva general de la experiencias de evaluación de la docencia en España, la valoración por parte de los implicados es negativa (Tejedor, 1993: 96- 98). Además de considerar que los implicados valoran de forma negativa este proceso, propone que con la elaboración de un buen diseño del instrumento utilizado que 
podría ser bien acogido por la comunidad universitaria. Así, la evaluación podría convertirse en una estrategia útil para la práctica docente empleada de modo eficaz a la buena programación de actividades formativas. Las técnicas utilizadas para la recogida de datos es una dificultad añadida de la evaluación de la docencia. La utilización de los instrumentos elaborados para este fin a nivel institucional, en la mayoría de las

ocasiones, no es eficaz.

Así pues, una de las mayores problemáticas en las evaluaciones de la docencia de los profesores universitarios, aunque la literatura la considera escasamente, está relacionada con el escaso presupuesto destinado para este cometido. Esto a su vez supone que tanto los medios materiales como personales sean escasos y limitados.

\section{Objetivo}

El presente trabajo forma parte de una refiriéndonos a la valoración otorgada por investigación más amplia en la que se analizan los resultados de la evaluación de la docencia en la UPSA. El estudio que presentamos en este artículo tiene como propósito analizar la información obtenida sobre la evaluación docente de los profesores de esta Universidad,

\section{Método}

Así la opción metodológica que mejor se adecua al trabajo propuesto para dar respuesta a los objetivos es la metodología cuantitativa, aunque los datos se van a tratar de forma descriptiva.

Siguiendo a Sabarriego Puig, M. y Bisquerra Alzina, R. (2004: 41), nos posicionamos en un paradigma positivista puesto que lo que pretende este trabajo es realizar un análisis de las variables, además, la metodología seguida en esta investigación es cuantitativa los alumnos a sus profesores y marcando diferencias por la categoría profesional del profesor evaluado. Recogemos los resultados de la evaluación tanto de las asignaturas teóricas como prácticas impartidas en todas las titulaciones de esta Universidad. 


\section{Muestra}

Las materias elegidas se presentan en dos modalidades (teóricas y prácticas), con instrumentos diferentes para cada una de ellas. La tabla nos permite observar cuántas son las materias que investiga el estudio. Se puede ver en la tabla siguiente:

\begin{tabular}{|l|l|l|}
\hline & $\begin{array}{l}\text { Número de } \\
\text { materias } \\
\text { prácticas }\end{array}$ & $\begin{array}{l}\text { Número de } \\
\text { materias } \\
\text { teóricas }\end{array}$ \\
\hline $\begin{array}{l}\text { Evaluación de } \\
\text { alumnos }\end{array}$ & 53 & 204 \\
\hline
\end{tabular}

Tabla 1: Número de materias seleccionadas para la muestra
La muestra de profesores que han sido evaluados por los alumnos es muy representativa, como se puede ver en la tabla que presentamos a continuación:

\begin{tabular}{|c|l|l|}
\hline № total de & o total de & \% de \\
profesores & profesores de & profesores \\
de todas los & los Centros y & que están \\
Centros y & Titulaciones & implicados \\
Titulaciones & implicados en & en el \\
de la UPSA & la evaluación & estudio \\
\hline 198 & 177 & $89,3 \%$ \\
\hline
\end{tabular}

Tabla 2: Población y muestra de profesores implicados en el estudio

\section{El instrumento}

El instrumento que se ha venido utilizando en la mayoría de Universidades Españolas, al igual que en la evaluación de la UPSA es el cuestionario.

El Comité de Calidad de la UPSA, utilizando diferentes cuestionarios realizados por otras Universidades Españolas (Salamanca, Deusto, A Coruña, Complutense,...), realizó un cuestionario para la evaluación del profesorado compuesto por 30 ítems para evaluar las materias teóricas y por 28 ítems para las materias de tipo práctico, con puntuaciones en una escala de tipo Likert desde 1 (mínimo acuerdo con la frase) hasta 5 (máximo acuerdo con la frase) incluyendo la opción NA (No Aplicable) para los casos en que no proceda la pregunta para la materia que se evalúa. Estos cuestionarios son cumplimentados tanto por los alumnos como por el/los profesor/es de la asignatura. En el caso del alumno, se le aconseja no responder al cuestionario si no ha asistido regularmente a clase.

Una vez elaborados los cuestionarios de teoría y práctica, antes de proceder al trabajo de campo para ser cumplimentados por las muestras a las que se dirigen, fue validado en el mismo contexto en el que se iba a realizar el estudio, con una muestra piloto de 1.200 cuestionarios obtenidos por muestreo aleatorio estratificado. Los análisis psicométricos realizados mostraron buenos índices de homogeneidad y discriminación de los ítems, así como fiabilidad y validez en las 
escalas finales. Para el estudio de fiabilidad, se ha empleado el coeficiente alfa de Cronbach, siendo este superior a 0,9 .

Martín Tabernero, M. ${ }^{\mathrm{a}}$ F. y Delgado Álvarez, C. (2002, 4-5), en un artículo sobre la evaluación de la docencia en esta Universidad, exponen que ambos cuestionarios, Teoría y Práctica, incluyen cinco factores de aspectos concretos de la función docente y un factor de valoración global del profesor/a, además de un apartado para la autovaloración del alumno.

El contenido de las dimensiones que compone el cuestionario son las siguientes:

- Planificación-Organización: ítems relacionados con la adecuación de la carga de trabajo de la asignatura, la materia explicada en clase, el desarrollo del programa, la adecuada preparación de las clases, etc...

- Metodología-Motivación:

ítems relacionados con el modo en que se motiva al alumnado por la asignatura, en que se incita a la reflexión sobre la utilidad de los conocimientos..., y otros temas de metodología como en qué medida se utiliza bibliografía adecuada, si señala lo importante del tema...

- Metodología-Desarrollo de las clases: ítems relacionados con el modo en que se imparte la asignatura, explicaciones ordenadas, consecución de los objetivos, uso de material apropiado, utilización de esquemas y/o gráficos explicativos,...

- Relación-Comunicación: ítems relacionados con la accesibilidad dentro y fuera de clase, clima de confianza, disposición para el diálogo...

- Evaluación: ítems relacionados con si el profesor dará criterios claros sobre la forma en que se realizará la evaluación, orientaciones sobre el tipo de examen, proporciona criterios claros para la elaboración de trabajos...

- Global: percepción global del profesor/a.

Se añaden al cuestionario siete preguntas adicionales que no valoran al profesor/a y que constituyen una autovaloración del alumno/a en los siguientes aspectos:

- - Pregunta 1 sobre regularidad de la asistencia a clase.

- - Preguntas 2, 3 y 4 sobre variables disposicionales del alumno/a hacia la asignatura: su objeto no es evaluar al profesor/a, sino la habilidad personal del alumno/a hacia los contenidos propios de la asignatura. Las variables evaluadas son: interés por la materia, motivación y deseo de continuar profundizando.

- - Pregunta 5 sobre la percepción de dificultad de la materia y las preguntas 6 y 7 sobre la exigencia del profesor/a y ritmo de la asignatura.

\section{Resultados}

A continuación se exponen, teniendo en cuenta la categoría profesional, las medias 
de las valoraciones que otorgan los alumnos de la docencia tanto de materias de carácter de la UPSA en los diferentes ítems de los teórico y de carácter práctico. cuestionarios elaborados para la evaluación

\begin{tabular}{|c|c|c|c|c|}
\hline ÍTEM & Catedrático & Titular & Adjunto & Ayudante \\
\hline Ítem 1: Desarrolla el programa. & 4,20 & 4,38 & 3,91 & 3,79 \\
\hline $\begin{array}{l}\text { Ítem 2: Tipo de práctica adecuado } \\
\text { a materia. }\end{array}$ & 3,98 & 4,46 & 3,89 & 3,75 \\
\hline $\begin{array}{l}\text { Ítem 3: Adecuada preparación de } \\
\text { las prácticas. }\end{array}$ & 4,11 & 4,44 & 3,78 & 3,55 \\
\hline $\begin{array}{l}\text { Ítem 4: Indica los objetivos de las } \\
\text { prácticas. }\end{array}$ & 3,97 & 4,37 & 3,66 & 3,25 \\
\hline $\begin{array}{l}\text { Ítem 5: Prácticas bien } \\
\text { estructuradas. }\end{array}$ & 3,87 & 4,21 & 3,49 & 3,39 \\
\hline $\begin{array}{l}\text { Ítem } 6 \text { : Sabe contagiar el interés } \\
\text { por las prácticas. }\end{array}$ & 2,77 & 4,25 & 3,05 & 2,65 \\
\hline Ítem 7: Incita a la reflexión. & 3,60 & 4,34 & 3,19 & 2,88 \\
\hline Ítem 8: Motiva a los alumnos. & 3,34 & 4,22 & 3,04 & 2,76 \\
\hline $\begin{array}{l}\text { Ítem 9: Las explicaciones se hacen } \\
\text { de forma ordenada. }\end{array}$ & 3,52 & 4,05 & 3,29 & 3,29 \\
\hline $\begin{array}{l}\text { Ítem 10: Indica lo importante de } \\
\text { cada práctica. }\end{array}$ & 3,25 & 3,92 & 3,18 & 2,87 \\
\hline $\begin{array}{l}\text { Ítem 11: Hace que el alumno se } \\
\text { cuestione aspectos de las } \\
\text { prácticas. }\end{array}$ & 3,35 & 4,22 & 3,14 & 2,83 \\
\hline $\begin{array}{l}\text { Ítem 12: Facilita explicaciones con } \\
\text { esquemas, gráficos... }\end{array}$ & 3,10 & 3,58 & 2,89 & 2,34 \\
\hline Ítem 13: Se le entiende bien. & 3,96 & 4,47 & 3,34 & 3,39 \\
\hline $\begin{array}{l}\text { Ítem 14: Las prácticas ayudan a } \\
\text { conseguir los objetivos del } \\
\text { programa. }\end{array}$ & 3,88 & 4,43 & 3,60 & 3,36 \\
\hline $\begin{array}{l}\text { Ítem 15: Es accesible para } \\
\text { consultar fuera de clase. }\end{array}$ & 3,36 & 3,67 & 3,79 & 3,48 \\
\hline $\begin{array}{l}\text { Ítem 16: Disposición para el } \\
\text { diálogo. }\end{array}$ & 3,66 & 4,08 & 3,85 & 3,68 \\
\hline $\begin{array}{l}\text { Ítem 17: Atiende las inquietudes } \\
\text { de los alumnos. }\end{array}$ & 3,89 & 4,01 & 3,69 & 3,54 \\
\hline $\begin{array}{l}\text { Ítem 18: Formula preguntas que } \\
\text { hacen reflexionar sobre las } \\
\text { prácticas. }\end{array}$ & 3,64 & 4,13 & 3,12 & 3,02 \\
\hline $\begin{array}{l}\text { Ítem 19: Fomenta el clima de } \\
\text { confianza en clase. }\end{array}$ & 4,05 & 3,96 & 3,60 & 3,49 \\
\hline $\begin{array}{l}\text { Ítem 20: Indica los criterios de } \\
\text { evaluación. }\end{array}$ & 3,52 & 4,13 & 3,31 & 3,11 \\
\hline
\end{tabular}




\begin{tabular}{|c|c|c|c|c|}
\hline $\begin{array}{l}\text { Ítem 21: Da orientaciones claras } \\
\text { sobre el tipo de examen. }\end{array}$ & 3,12 & 4,07 & 3,21 & 3,03 \\
\hline $\begin{array}{l}\text { Ítem 22: Comenta los trabajos y } \\
\text { actividades. }\end{array}$ & 3,60 & 4,31 & 3,66 & 3,43 \\
\hline $\begin{array}{l}\text { Ítem 23: Señala el modo de } \\
\text { corrección. }\end{array}$ & 3,04 & 3,72 & 3,00 & 2,86 \\
\hline $\begin{array}{l}\text { Ítem 24: Da orientaciones claras } \\
\text { para realizar los trabajos. }\end{array}$ & 2,58 & 4,02 & 3,29 & 3,09 \\
\hline $\begin{array}{l}\text { Ítem 25: Realiza seguimiento de } \\
\text { los trabajos. }\end{array}$ & 3,43 & 3,66 & 3,34 & 3,48 \\
\hline Ítem 26: Es un buen profesor. & 3,15 & 4,64 & 3,55 & 3,38 \\
\hline $\begin{array}{l}\text { Ítem } 27 \text { : Le gustaría que volviera } \\
\text { a ser su profesor. }\end{array}$ & 2,88 & 4,39 & 3,28 & 3,07 \\
\hline $\begin{array}{l}\text { Ítem 28: } \mathrm{Al} \text { profesor le gusta } \\
\text { enseñar esta asignatura. }\end{array}$ & 3,79 & 4,77 & 3,91 & 3,43 \\
\hline
\end{tabular}

Viendo la tabla 3 , se observa que en las valoraciones que otorgan los alumnos a sus profesores en las materias prácticas, los profesores titulares son los que obtienen las medias más altas; excepto en algunos de los aspectos del cuestionario como la accesibilidad fuera de clase, donde son los profesores adjuntos los que obtienen las medias más altas y fomentar la confianza en clase, donde son los catedráticos los mejor valorados. Sin embargo, son los profesores ayudantes los que obtienen las medias más bajas en la mayoría de las valoraciones de las materias prácticas que imparten, a excepción de aspectos como si se le entiende bien o si realiza seguimiento de los trabajos, donde son los profesores adjuntos los que tienen las medias más bajas y en otros como accesibilidad fuera de clase, disposición para el diálogo, orientaciones claras para los trabajos y les gustaría que volviera a ser su profesor, donde son los catedráticos los que obtienen las valoraciones más bajas.

\begin{tabular}{|l|c|c|c|c|}
\hline ÍTEM & Catedrático & Titular & Adjunto & Ayudante \\
\hline $\begin{array}{l}\text { Ítem 1: Desarrolla el } \\
\text { programa de la materia. }\end{array}$ & 4,03 & 4,31 & 4,04 & 2,58 \\
\hline $\begin{array}{l}\text { Ítem 2: Cantidad de la } \\
\text { materia adecuada. }\end{array}$ & 3,31 & 3,83 & 3,49 & 2,56 \\
\hline $\begin{array}{l}\text { Ítem 3: Adecuada } \\
\text { preparación. }\end{array}$ & 3,76 & 4,17 & 3,98 & 3,17 \\
\hline $\begin{array}{l}\text { Ítem 4: Indica los objetivos. } \\
\text { Ítem 5: La materia está bien }\end{array}$ & 3,49 & 3,99 & 3,84 & 2,77 \\
\hline estructurada. & 3,57 & 3,65 & 3,87 & 2,23 \\
\hline \begin{tabular}{l} 
Ítem 6: Adecuada carga de trabajo. \\
\hline Ítem 7: Incita a la reflexión.
\end{tabular} & 3,44 & 3,92 & 3,35 & 2,46 \\
\hline
\end{tabular}




\begin{tabular}{|c|c|c|c|c|}
\hline Ítem 8: Motiva a los alumnos. & 2,94 & 3,93 & 3,50 & 2,55 \\
\hline $\begin{array}{l}\text { Ítem 9: Las explicaciones son } \\
\text { adecuadas. }\end{array}$ & 3,42 & 4,17 & 3,30 & 2,63 \\
\hline $\begin{array}{l}\text { Ítem 10: Los trabajos facilitan el } \\
\text { aprendizaje de los alumnos. }\end{array}$ & 3,52 & 3,81 & 3,72 & 3,79 \\
\hline Ítem 11: Bibliografía adecuada. & 3,69 & 4,09 & 3,93 & 3,73 \\
\hline $\begin{array}{l}\text { Ítem 12: Indica lo importante del } \\
\text { tema. }\end{array}$ & 3,21 & 3,94 & 3,56 & 2,27 \\
\hline $\begin{array}{l}\text { Ítem 13: El alumno se cuestiona los } \\
\text { aspectos de clase. }\end{array}$ & 3,05 & 3,88 & 3,39 & 2,77 \\
\hline $\begin{array}{l}\text { Ítem 14: Facilita explicaciones con } \\
\text { esquemas, gráficos,... }\end{array}$ & 3,16 & 3,55 & 3,22 & 3,82 \\
\hline Ítem 15: Se le entiende bien. & 3,33 & 4,14 & 3,79 & 2,77 \\
\hline $\begin{array}{l}\text { Ítem 16: El desarrollo de las clases } \\
\text { ayuda a conseguir objetivos. }\end{array}$ & 3,36 & 3,75 & 3,69 & 2,56 \\
\hline $\begin{array}{l}\text { Ítem 17: Es accesible para consultar } \\
\text { fuera de clase. }\end{array}$ & 3,48 & 4,24 & 4,09 & 2,45 \\
\hline $\begin{array}{l}\text { Ítem 18: Disposición para el } \\
\text { diálogo. }\end{array}$ & 3,70 & 4,37 & 4,05 & 2,92 \\
\hline $\begin{array}{l}\text { Ítem 19: Atiende las inquietudes de } \\
\text { los alumnos. }\end{array}$ & 3,52 & 4,16 & 3,87 & 2,74 \\
\hline $\begin{array}{l}\text { Ítem 20: Hace reflexionar al } \\
\text { alumno. }\end{array}$ & 3,17 & 3,71 & 3,41 & 2,95 \\
\hline $\begin{array}{l}\text { Ítem 21: Fomenta el clima de } \\
\text { confianza en clase. }\end{array}$ & 3,35 & 4,20 & 3,86 & 2,61 \\
\hline $\begin{array}{l}\text { Ítem 22: Indica los criterios de } \\
\text { evaluación. }\end{array}$ & 3,07 & 3,72 & 3,65 & 2,47 \\
\hline $\begin{array}{l}\text { Ítem 23: Da orientaciones sobre el } \\
\text { examen. }\end{array}$ & 2,93 & 3,56 & 3,56 & 2,29 \\
\hline $\begin{array}{l}\text { Ítem 24: Comenta los trabajos y las } \\
\text { actividades. }\end{array}$ & 3,43 & 3,76 & 3,66 & 2,50 \\
\hline $\begin{array}{l}\text { Ítem 25: Señala el modo de } \\
\text { corrección. }\end{array}$ & 2,80 & 3,39 & 3,22 & 2,09 \\
\hline $\begin{array}{l}\text { Ítem 26: Da orientaciones claras } \\
\text { para realizar los trabajos. }\end{array}$ & 3,12 & 3,05 & 3,43 & 2,08 \\
\hline $\begin{array}{l}\text { Ítem 27: Hace seguimiento de los } \\
\text { trabajos. }\end{array}$ & 2,76 & 3,54 & 3,33 & 1,74 \\
\hline Ítem 28: Es un buen profesor. & 3,32 & 4,20 & 3,71 & 2,61 \\
\hline $\begin{array}{l}\text { Ítem 29: Le gustaría que volviera a } \\
\text { ser su profesor. }\end{array}$ & 3,01 & 3,78 & 3,37 & 2,17 \\
\hline $\begin{array}{l}\text { Ítem 30: } \mathrm{Al} \text { profesor le gusta } \\
\text { enseñar esta asignatura. }\end{array}$ & 3,68 & 4,61 & 4,28 & 3,52 \\
\hline
\end{tabular}

Tabla 4: Puntuaciones medias por categoría profesional en materias teóricas

También en las valoraciones de las materias los mejor valorados, o si facilita explicaciones teóricas, como puede verse en la tabla 4, con esquemas, donde son los ayudantes los son los profesores titulares los que obtienen que obtienen la media más alta. las medias más altas en la mayoría de los Sin embargo, vuelven a ser los profesores aspectos, excepto en ítems como si la materia ayudantes los que obtienen las medias más está bien estructurada o si da orientaciones bajas en la mayoría de aspectos valorados en claras en los exámenes, donde son los adjuntos las materias teóricas, excepto en si los trabajos 
facilitan el aprendizaje, si la bibliografía es adecuada y facilita explicaciones con esquemas, gráficos,... donde son los catedráticos los menos valorados.

Las opiniones de la muestra global se presentan siguiendo los cinco factores considerados en el cuestionario: Planificación y Organización, Metodología-desarrollo de las clases, Relación-comunicación, Evaluación y Valoración global.

\section{Factor 1: Planificación y Organización}

Los alumnos manifiestan acuerdo con el factor 1 de los cuestionarios. Las tablas 5 y 6 muestran que la media del factor 1 supera el 3, independientemente de la categoría profesional del profesor que imparte la materia. En el caso de la evaluación de las asignaturas prácticas (tabla 6), las materias impartidas por profesores titulares tienen una media más alta que las materias impartidas por el resto de profesores, aunque las medias son muy similares. Por otro lado, en la evaluación de las asignaturas teóricas (tabla 5), los alumnos muestran más acuerdo con la Planificación y la Organización que desarrollan aquellos profesores que pertenecen a la categoría profesional de titular y adjunto (medias más altas), aunque también las medias son muy similares. La diferencia más destacada es que los profesores ayudantes son los que obtienen una media más baja.

\section{Factor 2: Metodología y Motivación}

Los alumnos manifiestan acuerdo con el factor 2 de los cuestionarios. Las tablas 5 y 6 indican que la media supera el 3 en la mayoría de los casos. En el caso de la evaluación de las asignaturas prácticas (tabla $6)$, los alumnos muestran acuerdo con la Metodología y Motivación de la asignatura independientemente de la categoría profesional de sus profesores. Aunque ligeramente más alta, las materias impartidas por profesores titulares tienen una media más alta que las materias impartidas por el resto de profesores y los profesores ayudantes son los que obtienen la media más baja. Por otro lado, en la evaluación de las asignaturas teóricas (tabla 5), los alumnos muestran más acuerdo con la Metodología y Motivación que desarrollan aquellos profesores que pertenecen a la categoría profesional de titular y adjunto (medias más altas). La diferencia más destacada es con los profesores ayudantes y con los catedráticos, que obtienen las medias más bajas. Según los alumnos, los profesores catedráticos y los ayudantes, la categoría más alta y la más baja, son los que utilizan una metodología menos apropiada y menos les motivan en sus clases.

Factor 3: Metodología-desarrollo de las clases Los alumnos manifiestan acuerdo con el factor 3 de los cuestionarios. Las tablas $5 \mathrm{y}$ 6 señalan que la media del factor 3 supera el 3 en la mayoría de los casos. En el caso de la evaluación de las asignaturas prácticas (tabla 6), los alumnos muestran acuerdo en la Metodología-desarrollo de las clases, independientemente de la categoría profesional de sus profesores, las materias impartidas por profesores titulares tienen una media más alta que las materias impartidas por el resto de profesores y los profesores ayudantes 
son los que obtienen la media más baja. Por otro lado, en la evaluación de las asignaturas teóricas (tabla 5), los alumnos muestran más acuerdo con la Metodología-desarrollo de las clases que desarrollan aquellos profesores que pertenecen a la categoría profesional de titular y adjunto (medias más altas). La diferencia más destacada es con los profesores ayudantes que obtienen la media más baja.

\section{Factor 4: Relación-comunicación}

Los alumnos manifiestan acuerdo con el factor 4 de los cuestionarios. Las tablas 5 y 6 muestran que la media supera el 3 en la mayoría de los casos. En el caso de la evaluación de las asignaturas prácticas (tabla 6), los alumnos muestran acuerdo en el factor, independientemente de la categoría profesional de sus profesores. Ligeramente, las materias impartidas por profesores titulares tienen una media más alta que las materias impartidas por el resto de profesores y los profesores ayudantes son los que obtienen la media más baja. Por otro lado, en la evaluación de las asignaturas teóricas (tabla 5), los alumnos muestran más acuerdo con las materias impartidas por profesores que pertenecen a la categoría profesional

\section{Discusión}

La evaluación en la Universidad nos permite conocer los aspectos fuertes y los débiles de la institución, así como las áreas de mejora, el rendimiento académico logrado,... etc. La mayoría de estudios consideran que la evaluación de la docencia universitaria debería de titular y adjunto (medias más altas), la diferencia más significativa es con los profesores ayudantes que obtienen la media más baja.

\section{Factor 5: Evaluación}

Los alumnos manifiestan acuerdo con el factor 5 de los cuestionarios. Las tablas 5 y 6 señalan que la media supera el 3 en la mayoría de los casos. En el caso de la evaluación de las asignaturas prácticas (tabla 6), los alumnos muestran acuerdo en este factor independientemente de la categoría profesional de sus profesores. Aunque las medias son muy próximas, las materias impartidas por profesores titulares tienen una media más alta que las materias impartidas por el resto de profesores y los profesores catedráticos son los que obtienen la media más baja. Por otro lado, en la evaluación de las asignaturas teóricas (tabla 5), los alumnos muestran más acuerdo con las materias impartidas por profesores que pertenecen a la categoría profesional de titular y adjunto (medias más altas), la diferencia más significativa es con los profesores ayudantes, que obtienen la media más baja.

formar parte de un programa más amplio referido a la institución universitaria, porque se trata de un fenómeno complejo, enfocado a conseguir una mejora de la enseñanza.

Se concluye que la importancia de la evaluación de la docencia puede venir dada, o 
bien en función de la utilidad de los resultados obtenidos, o bien en la propia mejora que genera.

Aun cuando los problemas o inconvenientes de la evaluación docente son diversos, al menos las investigaciones revelan que es importante tener claro qué queremos evaluar, exigiendo coherencia entre las dimensiones propuestas para ser evaluadas con los objetivos de la evaluación, además de elegir bien el sistema de evaluación, para evitar la resistencia del profesorado a su evaluación.

En cuanto a las fuentes de la evaluación, algunos estudios rechazan la de evaluación por colegas, y consideran de mayor utilidad la evaluación por los alumnos, dado que son los mejores observadores del proceso de enseñanza-aprendizaje, aunque pueden darse sesgos debido a diversos factores (calificaciones que otorga el profesor, la reputación del mismo, su dedicación, teorías del buen profesor, tipo de materia, años de experiencia, categoría profesional o rango del profesor, obligatoriedad de las materias, características personales del profesor, niveles altos de trabajo y dificultad, interés previo del alumno, razón para elegir, variables del profesor y variables del estudiante, características del curso y contexto, el tipo de test utilizado y si los que valoran son alumnos o ex alumnos). Si tenemos en cuenta uno de los sesgos que se señalan en la mayoría de estudios relacionados con el tema, la categoría profesional, en este trabajo se encuentran diferencias, pero no demasiado significativas en la mayoría de los casos.
Tanto en asignaturas prácticas como en teóricas, los alumnos manifiestan estar de acuerdo con los ítems del cuestionario, independientemente de la categoría profesional del profesor. Sin embargo, en las prácticas valoran mejor, en muchos de los aspectos, a los profesores titulares y con medias más bajas a los profesores ayudantes. Las materias teóricas que están impartidas por los profesores ayudantes son las peor valoradas. Quizá la falta de experiencia profesional pueda ser la causa por la que los alumnos consideren peor profesor al profesor ayudante respecto a los profesores de otras categorías.

En el análisis de la disposición del alumno hacia la materia, en función de la categoría profesional del profesor, pone de manifiesto que los que cursan asignaturas impartidas por profesores titulares tienen una disposición ligeramente más alta que el resto de alumnos. Sin embargo manifiestan menor disposición cuando los profesores son ayudantes. En las materias teóricas, los alumnos están más dispuestos en las impartidas por profesores titulares y adjuntos, y menos en las impartidas por profesores ayudantes.

Los alumnos en materias prácticas perciben mayor dificultad, exigencia y ritmo cuando son impartidas por profesores titulares y adjuntos, con puntuaciones ligeramente más altas, que cuando son impartidas por los profesores del resto de categorías profesionales, incluídos los profesores catedráticos. Según los alumnos, las materias teóricas de mayor dificultad, ritmo y exigencia son las impartidas por 
profesores ayudantes. Este dato puede ser relevante y curioso para profundizar en otras investigaciones sobre el tema.

Puede parecernos sorprendente, pues la hipótesis que nos planteábamos de partida era la contraria, a mayor categoría profesional más dificultad, exigencia, ritmo de las asignaturas, así como mayor disposición de los alumnos hacia las materias y también más valoración en los ítems de evaluación docente. Pero en este estudio no vemos que esta relación sea clara en todos los casos. Por lo que no podemos afirmar que a mayor categoría profesional del profesor los alumnos consideren que este sea mejor o más competente. Lo que si podemos afirmar, en líneas generales, es que en este estudio los profesores de la Universidad Pontificia mejor considerados por sus alumnos son los titulares, no los catedráticos, así como los peor valorados los ayudantes.

Numerosos trabajos recogen ideas sobre el buen profesor, algunas de ellas mediante la recopilación de una serie de características y otras mediante una serie de comportamientos que debería llevar a cabo el profesor universitario en su actividad docente, entre ellos Álvarez; García y Gil (1999a: 447), De la Orden (1990: 17), Kulik y McKeachie (1975: 219), Wotruba y Wright (1974: 656), McKeachie (1983: 37- 39), Debesse y Mialaret (1980: 124), Gallego (1988: 127), Tejedor y Montero (1990: 263). Sin embargo ninguno de estos autores incluye en sus planteamientos la relación entre categoría profesional y ser mejor profesor. Se refieren fundamentalmente a características relacionadas con el desarrollo de las clases, características de personalidad del profesor, relaciones interpersonales y evaluación. Aunque muchos de ellos también consideran importante las cuestiones relacionadas con los métodos de enseñanza y con la participación y necesidades de los alumnos. Otros, aunque en menor medida, consideran importante destacar la apariencia del profesor y la adecuada carga docente y exigencia discente. Por lo que la categoría profesional no la consideran importante para la calidad del desempeño docente del profesor universitario.

\section{Referencias}

Agència per a la Qualitat del Sistema docencia mejor evaluada por los alumnos en Universitari a Catalunya (2002). Marco las diferentes áreas de enseñanza universitaria, general de la evaluación del profesorado, Revista española de pedagogía, 214, 445-465. Barcelona.

Cano, E., \& Ion, G. (2012). Prácticas

Álvarez Rojo, V., García Jiménez, E., \& evaluadoras en las Universidades Catalanas: Gil Flores, J. (1999). Características de la Hacia un modelo centrado en competencias. 
Revista Estudios sobre Educación, 22, 155177.

Casillas Martín, S. (2006). Las percepciones de los alumnos sobre el desempeño docente de sus profesores en función de distintas variables. Revista Ciencias de la Educación, 208, 519-539.

Casillas Martín, S. (2007). Competencias y tareas específicas del profesor universitario. Revista Papeles Salmantinos de Educación, 9, 313-326.

Casillas Martín, S. (2008). El proceso de evaluación de la docencia en la Universidad. Revista Papeles Salmantinos de Educación, 11, 89-105.

Casillas Martín, S., \& Cabezas González, M. (2009). La evaluación del profesorado universitario. Revista Galego-Portuguesa de Psicoloxía e Educación, 17(1,2), 75-84.

Casillas Martín, S., \& Cabezas González, M. (2010). Distintas concepciones sobre la buena docencia universitaria. Revista Ciencias de la Educación, 223, 351-369.

De la Orden, A. (1990). Evaluación, selección y promoción del profesorado universitario. Revista Complutense de Educación, 1(1), 1129.

De Miguel Díaz, M. (1998). La evaluación del profesor universitario: Criterios y propuestas para mejorar la función docente. Revista de Educación, 315, 67-85.

De Miguel Díaz, M. (2003). Nuevos retos en el ámbito de la educación. Ponencia presentada en: XI Congreso sobre modelos de investigación educativa, Granada, (25- 27 de septiembre), (pp. 1-19).

Debesse, M., \& Mialaret, G. (1980). La función docente. Barcelona: OIKOS- TAU.

Gallego, P. (1988). El perfeccionamiento del profesorado: Un proyecto en clase formativa y multidimensional. En las Actas del II Congreso mundial Vasco, Servicio Central de Publicaciones de Gobierno Vasco, TOMO IV (pp. 124- 129).

Gil-Flores, J. (2012). La evaluación del aprendizaje en la Universidad según la experiencia de los estudiantes. Revista Estudios sobre Educación, 22, 133-153.

Guerrero Barona, E., \& Vicente Castro, F. (1999). Estudios sobre la importancia del factor humano en la realización profesional y personal: satisfacciones e insatisfacciones de la profesión docente universitaria. Revista electrónica interuniversitaria de formación del profesorado, 2(1), 579-586. Extraído el 10 de enero de 2013, de: http://www.uva.es/ aufop/publica/revelfop/99-v2n1.htm.

Jornet, J. M. (1993). Enfoques de la evaluación universitaria. En las Actas III Jornadas 
Nacionales de Didáctica Universitaria Sabarriego Puig, M., \& Bisquerra Alzina, R. "Evaluación y desarrollo profesional". (2004). Fundamentos metodológicos de la Ponencias y réplicas. Universidad las Palmas investigación educativa. En Bisquerra Alzina, de Gran Canaria (pp. 63-83).

R. (Coord), Metodología de la investigación educativa (pp. 17-49). Madrid: La Muralla.

Kulik, J. A, \& Mckeachie, W. J. (1975). The evaluation of teachers in higher education. Tascón Trujillo, C. (1998). Calidad de vida Review of Research in Education, 3, 210- 240. laboral en las instituciones universitarias: sugerencias en torno a la evaluación de L.R.U. (Ley Orgánica 11/ 1983, de 25 de la calidad en la Universidad. En las Actas Agosto, de Reforma Universitaria -BOE del Congreso Nacional: la formación del núm. 209, de 1 de Septiembre). profesorado. Evaluación y calidad (pp. 817827). Las Palmas de Gran Canaria: Servicio

Martín Tabernero, M. a F., \& Delgado de publicaciones de la Universidad de Las Álvarez, C. (2002). Análisis estadístico de la Palmas de Gran Canaria. percepción de la docencia en estudiantes de psicología. Publicaciones UPSA, "Temas de Psicología IX".

Mateo, J. (1990). Función docente y demanda Tejedor Tejedor, F. J., \& García-Valcárcel, A. (1996). La evaluación de la calidad de la docencia universitaria (en el marco de la evaluación institucional) desde la perspectiva discente. Revista Española de Pedagogía, 186(XLVIII), 319- 326.

de los alumnos. En: Tejedor Tejedor, F. J., \& Rodríguez Diéguez (Eds.), Evaluación Educativa II. Evaluación Institucional. Mckeachie, W. J. (1983). The role of Faculty Fundamentos Teóricos y Aplicaciones Evaluation in Enhancing College Teaching, Prácticas (pp. 93- 122). Documentos National Forum. Phi Kappa Journal, 63(2), Didácticos 157, IUCE, Universidad de $37-39$. Salamanca.

Rodríguez Espinar, S. (1998). Evaluación del Tejedor Tejedor, F. J. (1993). Experiencias profesorado y titulaciones universitarias. En las Actas del Congreso Nacional: La formación del profesorado. Evaluación y Calidad, (pp. 211- 235). Las Palmas de Gran Canaria: Servicio de Publicaciones Universidad Las Palmas de Gran Canaria. Españolas de evaluación de la enseñanza Universitaria y nuevas perspectivas. En las Actas de las III Jornadas Nacionales de didáctica Universitaria (pp. 87-109). Las Palmas de Gran Canaria: Universidad Las Palmas de Gran Canaria. 
Tejedor Tejedor, F. J. (2003). Un modelo evaluación del profesorado universitario. de evaluación del profesorado universitario. Revista Española de Pedagogía, 186, 259-279. RIE, 21(1), 157- 181.

Wotruba, T. R., \& Wright, P. L. (1974).

Tejedor Tejedor, F. J. (2009). Evaluación How to develop a teacher-rating instrument: del profesorado universitario: Enfoque A research approach. Journal of Higher metodológico y algunas aportaciones de Education, 46(6), 653- 663. http://dx.doi. la investigación. Revista Estudios sobre org/10.2307/1979060

Educación, 16, 79-102.

Zabalza Beraza, M. A. (1990). Evaluación Tejedor Tejedor, F. J., \& Montero, L. (1990). orientada al perfeccionamiento. Revista Indicadores de la Calidad Docente para la Española de Pedagogía, 186, 295-317.

\section{Notes}

${ }^{1}$ Este trabajo ha contado con el apoyo y la financiación de la Universidad Pontificia de Salamanca (UPSA) en su marco de la evaluación del profesorado para la mejora de la calidad de la educación superior. 